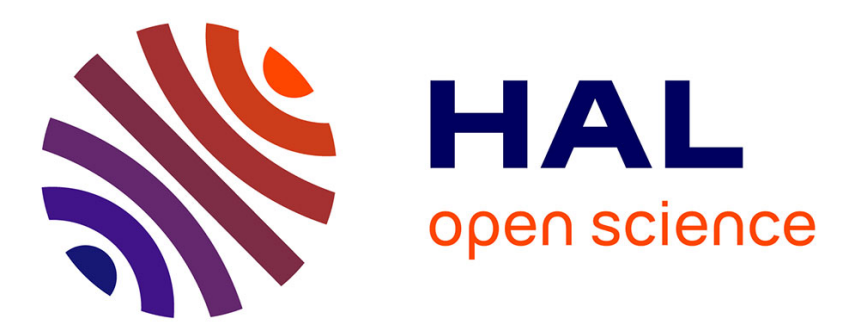

\title{
Operator calculus and invertible Clifford Appell systems: theory and application to the n-particle fermion algebra René Schott, George Stacey Staples
}

\section{To cite this version:}

René Schott, George Stacey Staples. Operator calculus and invertible Clifford Appell systems: theory and application to the n-particle fermion algebra. Infinite Dimensional Analysis, Quantum Probability and Related Topics, 2013, 16 (1), 10.1142/S0219025713500070 . hal-00586381

\section{HAL Id: hal-00586381 \\ https://hal.science/hal-00586381}

Submitted on 15 Apr 2011

HAL is a multi-disciplinary open access archive for the deposit and dissemination of scientific research documents, whether they are published or not. The documents may come from teaching and research institutions in France or abroad, or from public or private research centers.
L'archive ouverte pluridisciplinaire HAL, est destinée au dépôt et à la diffusion de documents scientifiques de niveau recherche, publiés ou non, émanant des établissements d'enseignement et de recherche français ou étrangers, des laboratoires publics ou privés. 


\title{
Operator calculus and invertible Clifford Appell systems: theory and application to the n-particle fermion algebra
}

\author{
René Schott, G. Stacey Staples ${ }^{\dagger}$
}

\begin{abstract}
Motivated by evolution equations on Clifford algebras and illustrated with the $n$-particle fermion algebra, a theory of invertible leftand right-Appell systems is developed for Clifford algebras of an arbitrary quadratic form. This work extends and clarifies the authors' earlier work on Clifford Appell systems, operator calculus, and operator homology/cohomology. A direct connection is also shown between blade factorization algorithms and the construction of Appell systems in these algebras.
\end{abstract}

\section{Introduction}

Over the years, Clifford algebras have proven advantageous for a broad range of applications in physics and engineering. More recently, their utility in implementing quantum algorithms has been shown [4], [12] Some familiar examples of Clifford algebras include the algebra of quaternions and the $n$-particle fermion creator/annihilator algebra.

Appell systems can be interpreted as polynomial solutions of generalized heat equations. In probability theory, they are also used to obtain non-central limit theorems. Their analogues have been defined on Lie groups [8], the Schrödinger algebra [7], and quantum groups [6]. Clifford Appell systems are natural objects of interest for constructing solutions of Clifford evolution equations.

The current authors first defined general Appell systems within a Clifford algebra of arbitrary signature in [14]. The operator calculus (OC) appearing in that preliminary work was subsequently used

*IECN and LORIA Université Henri Poincaré-Nancy I, BP 239, 54506 Vandoeuvre-lèsNancy, France, email: schott@loria.fr

${ }^{\dagger}$ Department of Mathematics and Statistics, Southern Illinois University Edwardsville, Edwardsville, IL 62026-1653, USA, email: sstaple@siue.edu 
in a treatment of operator homology and cohomology in Clifford algebras [18]. More recently, OC methods were extended to commutative subalgebras of the Grassmann exterior algebra referred to herein as "zeon" algebras and used to give an OC formulation of partitiondependent stochastic measures [19].

The authors' OC approach is implicit in a number of earlier works in graph theory (cf. [22], [20]). Operator calculus on zeon algebras provides the common context for relating graph theory and quantum random variables [15]. Moreover, the OC approach was advantageous in developing a graph-theoretic construction of stochastic integrals in Clifford algebras of arbitrary signature [21].

The goal of the current work is to clarify the role of operator calculus in solving Clifford Appell systems. Geometric interpretations are given for combinatorially-defined operators. Notions of invertible leftand right-Appell systems are developed, and the role of blade factorization in constructing Appell systems is clarified.

\section{Essential background}

Presented here are some concepts, definitions, and notational conventions used throughout subsequent sections.

\section{$2.1 \quad$ Appell systems}

Following the formalism of Feinsilver, Kocik, and Schott [7], the space of polynomials with degree not exceeding $n$ can be considered as the space of solutions, $\mathcal{Z}_{n}$, to the equation $D^{n+1} \psi=0$, where $D$ is the differentiation operator. In this context, an Appell system is a sequence of nonzero polynomials satisfying two conditions:

i. $\psi_{n} \in \mathcal{Z}_{n}, \forall n \geq 0$, and

ii. $D \psi_{n}=\psi_{n-1}, \forall n \geq 1$.

A simple example of an Appell system is to define $\psi_{n}=x^{n} / n$ ! with $D=d / d x$. Other examples of Appell systems include shifted moment sequences

$$
\psi_{n}(x)=\int_{-\infty}^{\infty}(x+y)^{n} p(d y)
$$

where $p$ is a probability measure on $\mathbb{R}$ with all moments finite. This includes the Hermite polynomials,

$$
H_{n}(x)=\frac{1}{\sqrt{2 \pi t}} \int_{-\infty}^{\infty}(x+y)^{n} e^{-y^{2} / 2 t} d y, t>0,
$$


for the Gaussian case. These polynomials are the solutions of the heat equation

$$
\partial_{t} f(x, t)=\frac{1}{2} \partial_{x}^{2} f(x, t)
$$

with $\lim _{t \rightarrow 0} f(x, t)=x^{n}$.

More generally, for any operator $\mathcal{A}$, one sets

$$
\mathcal{Z}_{n}=\left\{\psi: \mathcal{A}^{n+1} \psi=0\right\}
$$

for $n \geq 0$, and defines an $\mathcal{A}$-Appell system as a sequence of nonzero functions $\left\{\psi_{0}, \psi_{1}, \ldots, \psi_{n}, \ldots\right\}$ satisfying

i. $\psi_{n} \in \mathcal{Z}_{n}, \forall n \geq 0$, and

ii. $\mathcal{A} \psi_{n}=\psi_{n-1}$, for $n \geq 1$.

The system of embeddings $\mathcal{Z}_{0} \subset \mathcal{Z}_{1} \subset \mathcal{Z}_{2} \subset \cdots$ is referred to as a canonical $\mathcal{A}$-Appell system decomposition.

\subsection{Clifford algebras}

For convenience, the following notation is recalled for the commutator and anti-commutator of algebraic elements, respectively:

$$
\begin{gathered}
{[x, y]:=x y-y x} \\
{[x, y]_{+}:=x y+y x .}
\end{gathered}
$$

Let $n \in \mathbb{N}$, and let $p, q$ be nonnegative integers such that $p+q=n$. Let $V$ be a real inner product space with orthonormal basis $\left\{\mathbf{e}_{i}\right.$ : $1 \leq i \leq n\}$. The Clifford algebra of signature $(p, q)$, denoted $\mathcal{C} \ell_{p, q}$, is defined as the real associative algebra generated by the vectors $\left\{\mathbf{e}_{i}\right\}$, along with the unit scalar 1 subject to the following multiplication rules:

$$
\left[\mathbf{e}_{i}, \mathbf{e}_{j}\right]_{+}= \begin{cases}2 & \text { if } 1 \leq i=j \leq p, \\ -2 & \text { if } p+1 \leq i=j \leq n, \\ 0 & \text { if } i \neq j .\end{cases}
$$

Denote the $n$-set $\{1, \ldots, n\}$ by $[n]$, and denote the associated power set by $2^{[n]}$. Adopting multi-index notation, the ordered product of generators is denoted

$$
\prod_{i \in I} \mathbf{e}_{i}=\mathbf{e}_{I},
$$

for any subset $I \subseteq[n]$, also denoted $I \in 2^{[n]}$.

These products of generators are referred to as basis blades for the algebra. The grade of a basis blade is defined to be the cardinality of 
its multi-index. An arbitrary element $u \in \mathcal{C} \ell_{p, q}$ has a canonical basis blade decomposition of the form

$$
u=\sum_{I \subseteq[n]} u_{I} \mathbf{e}_{I},
$$

where $u_{I} \in \mathbb{R}$ for each multi-index $I$. The grade- $k$ part of $u \in \mathcal{C} \ell_{p, q}$ is then naturally defined by

$$
\langle u\rangle_{k}:=\sum_{|I|=k} u_{I} \mathbf{e}_{I}
$$

It finally becomes evident that $\mathcal{C} \ell_{p, q}$ has a canonical vector space decomposition of the form

$$
\mathcal{C} \ell_{p, q}=\bigoplus_{k=0}^{n}\left\langle\mathcal{C} \ell_{p, q}\right\rangle_{k}
$$

An arbitrary element $u \in \mathcal{C} \ell(Q)$ is said to be homogeneous of grade $k$ if

$$
\begin{gathered}
\langle u\rangle_{k} \neq 0, \text { and } \\
\langle u\rangle_{\ell}=0, \forall \ell \neq k .
\end{gathered}
$$

As the degree of a polynomial refers to the maximal exponent appearing in terms of the polynomial, an arbitrary multivector $u \in \mathcal{C} \ell(Q)$ is said to be heterogeneous of grade $k$ if

$$
\begin{gathered}
\langle u\rangle_{k} \neq 0, \text { and } \\
\langle u\rangle_{\ell}=0, \ell>k .
\end{gathered}
$$

Recall the ordering symbol $\theta_{i j}$ defined by

$$
\theta_{i j}= \begin{cases}1 & \text { if } i<j \\ 0 & \text { otherwise }\end{cases}
$$

and let elements of multi-indices be expressed as $I=\left\{I_{1}, \ldots, I_{|I|}\right\}$.

The multi-index product signature map $\vartheta: 2^{[n]} \times 2^{[n]} \rightarrow\{ \pm 1\}$ is defined by

$$
\vartheta(I, J)=\exp \left(\imath \pi\left(|\{I \cap J \cap(p, n]\}|+\sum_{k=1}^{|J|} \sum_{\ell=1}^{|I|} \theta_{j_{k} i_{\ell}}\right)\right) .
$$

The product of basis blades in $\mathcal{C} \ell_{p, q}$ is now correctly given by

$$
\mathbf{e}_{I} \mathbf{e}_{J}=\vartheta(I, J) \mathbf{e}_{I \triangle J},
$$

where $\triangle$ represents the set symmetric difference operator, i.e., $I \triangle J:=$ $(I \cup J) \backslash(I \cap J)$. 
Example 2.1. Specific examples of Clifford algebras include the following:
i. $\mathcal{C} \ell_{0,1} \simeq \mathbb{C}$
ii. $\mathcal{C} \ell_{0,2} \simeq \mathbb{H}$
iii. $\mathcal{C} \ell_{3,0} \simeq$ APS algebra of physical space
iv. $\mathcal{C} \ell_{1,3} \simeq \mathrm{STA}$ spacetime algebra
v. $\mathcal{C} \ell_{n, 0} \simeq\{n$-particle fermion Fock space $\}$
vi. $\mathcal{C} \ell_{n, n} \simeq\{n$-particle fermion creator/annihilator algebra $\}$

The principal motivation for discussing Appell systems in the context of Clifford algebras is obtaining solutions to Clifford evolution equations. The evolution equations detailed herein describe discrete Clifford-valued processes taking the form of sequences $\left(u_{k}\right)_{k>0}$. The meaning of $\partial_{t}$ is thus understood by

$$
\partial_{t} u(k)=\Delta u=u_{k+1}-u_{k} .
$$

One straightforward example is

$$
\partial_{t} u=\Lambda u
$$

where $u=u(t) \in \mathcal{C} \ell(Q)$ and $\Lambda$ is an operator acting as generalized differentiation, or combinatorial lowering. Similarly, one can have an equation of the form

$$
\partial_{t} u=\Xi u,
$$

where $\Xi$ is a generalized integral, or combinatorial raising. Considering discrete processes of sums of raising and lowering operators gives

$$
\partial_{t} u=(\Lambda+\Xi) u,
$$

which can be regarded as a random walk on a directed hypercube [16].

It will be shown in a subsequent section that all solutions of the Clifford heat equation

$$
\partial_{t} u=\Lambda^{2} u
$$

are constant.

Before constructing Appell systems in Clifford algebras, an operator calculus for Clifford algebras must be discussed. Within the context of this calculus, the construction of Appell systems will be natural.

\section{Operator calculus in Clifford algebras}

The motivation for development of Clifford operator calculus is based on polynomial operator calculus. To begin, raising and lowering operators are defined naturally in terms of polynomial differentiation and 
integration operators on Clifford multivectors regarded as polynomials in anticommuting variables.

In this formulation, the generators $\left\{\mathbf{e}_{i}\right\}$ of $\mathcal{C} \ell_{p, q}$ are fixed a priori, and are regarded both as vectors in the Clifford algebra sense and as anti-commuting variables in the polynomial sense. In this context, the Clifford algebra $\mathcal{C} \ell_{p, q}$ is naturally regarded as an algebra of "Clifford polynomials" as well as Clifford multivectors.

After an initial discussion based on the notion of Clifford polynomials, the construction is subsequently generalized to Clifford algebras associated with arbitrary, non-degenerate quadratic forms. In this construction, no such generating set need be fixed, as a basis-free definition of the Clifford algebra over $V$ is given in terms of a non-degenerate quadratic form $Q$. The notation for this more generally constructed algebra is $\mathcal{C} \ell(Q)$. In this setting, the combinatorially and algebraically defined raising and lowering operators are more naturally envisioned in terms of the exterior product and left-contraction operators.

Analogous to polynomial differentiation and integration, the following operators are defined in the Clifford algebra context.

Definition 3.1. Let $I \in 2^{[n]}$ be an arbitrary multi-index. For $1 \leq$ $j \leq n$, define the $j^{\text {th }}$ Clifford differentiation operator $\partial / \partial \mathbf{e}_{j}$ by linear extension of

$$
\frac{\partial}{\partial \mathbf{e}_{j}} \mathbf{e}_{I}= \begin{cases}\vartheta(\{j\}, I) \mathbf{e}_{I \backslash\{j\}} & \text { if } j \in I, \\ 0 & \text { otherwise. }\end{cases}
$$

Definition 3.2. The Clifford integrals are defined by

$$
\begin{gathered}
\left\{\mathrm{d} \mathbf{e}_{i}, \mathrm{~d} \mathbf{e}_{j}\right\}=0 \text { for } i \neq j \\
\int \mathrm{d} \mathbf{e}_{j}=\mathbf{e}_{j}, \\
\iint \mathrm{d} \mathbf{e}_{i} \mathrm{~d} \mathbf{e}_{j}=\int \mathbf{e}_{i} \mathrm{~d} \mathbf{e}_{j}= \begin{cases}\mathbf{e}_{j} \mathbf{e}_{i} & \text { if } i \neq j \\
1 & \text { if } 1 \leq i=j \leq p \\
-1 & \text { if } p+1 \leq i=j \leq p+q\end{cases}
\end{gathered}
$$

so that

$$
\int \mathbf{e}_{I} \mathrm{~d} \mathbf{e}_{j}= \begin{cases}\vartheta(\{j\}, I) \mathbf{e}_{I \cup\{j\}} & \text { if } j \notin I \\ \vartheta(\{j\}, I) \mathbf{e}_{I \backslash\{j\}} & \text { if } j \in I .\end{cases}
$$

These polynomial operators induce combinatorial raising and lowering operators by which Clifford monomials (blades) are "raised" from grade $k$ to grade $k+1$ or "lowered" from grade $k$ to grade $k-1$. These raising and lowering operators can also be regarded as fermion creation and annihilation operators in the sense of quantum mechanics. 
Definition 3.3. For each $1 \leq j \leq n$, define the $j^{\text {th }}$ (left) raising operator $R_{j}$ by linear extension of

$$
R_{j} \mathbf{e}_{I}=\left\langle\int \mathbf{e}_{I} \mathrm{~d} \mathbf{e}_{j}\right\rangle_{|I|+1}=\mathbf{e}_{j} \wedge \mathbf{e}_{I}
$$

Define the $j^{\text {th }}$ (left) lowering operator $D_{j}$ by linear extension of

$$
D_{j} \mathbf{e}_{I}=\frac{\partial}{\partial \mathbf{e}_{j}} \mathbf{e}_{I}=\left\langle\mathbf{e}_{j} \mathbf{e}_{I}\right\rangle_{|I|-1} .
$$

Remark 3.4. Note that one can similarly define right raising and lowering operators. Indeed, these are the operators appearing in the authors' preliminary work [14]. The formulation involving left lowering and raising was considered in the authors' subsequent work [18]. For the remainder of the current work, all lowering and raising operators are regarded from the left unless specified otherwise.

Example 3.5. In the Clifford algebra $\mathcal{C} \ell_{2,2}$ the raising and lowering operators act in the following manner:

$$
\begin{gathered}
D_{1} \mathbf{e}_{\{1,2,3\}}=\mathbf{e}_{\{2,3\}} \\
R_{2} \mathbf{e}_{\{1,3\}}=-\mathbf{e}_{\{1,2,3\}} .
\end{gathered}
$$

The Clifford algebra $\mathcal{C} \ell_{p, q}$ admits a number of involution automorphisms, including reversion. The reversion of an element $u$ is denoted by $\tilde{u}$ and is defined by

$$
\tilde{u}:=\sum_{k=0}^{n}(-1)^{k(k-1) / 2}\langle u\rangle_{k} .
$$

The action of this automorphism is seen by reversing the order of vectors appearing in basis blades of $u$.

Reversion is useful in expressing the Euclidean inner product, defined on $\mathcal{C} \ell_{p, q}$, by

$$
\langle u, v\rangle:=\langle\tilde{u} v\rangle_{0}=\sum_{I \in 2^{[n]}} u_{I} v_{I} .
$$

Another inner product induced by the quadratic form associated with $\mathcal{C} \ell_{p, q}$ will also be useful in the discussion of Clifford operator calculus.

Letting $Q$ denote the following quadratic form on the vector space $V$ spanned by the generators of $\mathcal{C} \ell_{p, q}$ :

$$
Q(\mathbf{x})=x_{1}{ }^{2}+\cdots+x_{p}{ }^{2}-x_{p+1}{ }^{2}-\cdots-x_{n}{ }^{2},
$$

the algebra $\mathcal{C} \ell_{p, q}$ is also denoted by $\mathcal{C} \ell(Q)$. More generally, $Q$ will be considered any non-degenerate quadratic form on the vector space $V$. 
Associate with $Q$ the symmetric bilinear form

$$
\langle\mathbf{x}, \mathbf{y}\rangle_{Q}=\frac{1}{2}[Q(\mathbf{x}+\mathbf{y})-Q(\mathbf{x})-Q(\mathbf{y})],
$$

and extend to simple $k$-vectors in $\bigwedge^{k} V$ by

$$
\left\langle\mathbf{x}_{1} \wedge \mathbf{x}_{2} \wedge \cdots \wedge \mathbf{x}_{k}, \mathbf{y}_{1} \wedge \mathbf{y}_{2} \wedge \cdots \wedge \mathbf{y}_{k}\right\rangle_{Q}=\operatorname{det}\left\langle\mathbf{x}_{i}, \mathbf{y}_{j}\right\rangle_{Q}
$$

This inner product extends linearly to all of $\bigwedge^{k} V$ and by orthogonality to $\wedge V$.

The $Q$-inner product and exterior product extend to $\mathcal{C} \ell(Q)$ via the canonical vector space isomorphism. The left contraction operator is defined by (cf. [13, Chapter 14])

$$
\begin{gathered}
\mathbf{x}\lrcorner \mathbf{y}=\langle\mathbf{x}, \mathbf{y}\rangle_{Q} \forall \mathbf{x}, \mathbf{y} \in V ; \\
\mathbf{x}\lrcorner(u \wedge v)=(\mathbf{x}\lrcorner u) \wedge v+\hat{u} \wedge(\mathbf{x}\lrcorner v), \forall u, v \in \bigwedge V, \mathbf{x} \in V ; \\
(u \wedge v)\lrcorner w=u\lrcorner(v\lrcorner w), \forall u, v, w \in \bigwedge V .
\end{gathered}
$$

In particular, left and right contraction are dual to the exterior product and satisfy the following:

$$
\begin{aligned}
\langle u\lrcorner v, w\rangle_{Q} & =\langle v, \tilde{u} \wedge w\rangle_{Q}, \\
\left\langle u\llcorner v, w\rangle_{Q}\right. & =\langle u, w \wedge \tilde{v}\rangle_{Q} .
\end{aligned}
$$

Of particular importance, the exterior product and left contraction act as combinatorial raising and lowering operators in $\mathcal{C} \ell_{p, q}$ :

$$
\mathbf{e}_{j} \wedge \mathbf{e}_{I}=\left\langle\mathbf{e}_{j} \mathbf{e}_{I}\right\rangle_{|I|+1}= \begin{cases}\vartheta(\{j\}, I) \mathbf{e}_{I \cup\{j\}} & \text { if } j \notin I, \\ 0 & \text { otherwise; }\end{cases}
$$

and

$$
\left.\mathbf{e}_{j}\right\lrcorner \mathbf{e}_{I}=\left\langle\mathbf{e}_{j} \mathbf{e}_{I}\right\rangle_{|I|-1}= \begin{cases}\vartheta(\{j\}, I) \mathbf{e}_{I \backslash\{j\}} & \text { if } j \in I, \\ 0 & \text { otherwise. }\end{cases}
$$

It is now instructive to define the lowering operators in terms of contractions. In particular, the $j^{\text {th }}$ left lowering operator in $\mathcal{C} \ell_{p, q}$ is given by

$$
\left.D_{j} \mathbf{e}_{I}=\frac{\partial}{\partial \mathbf{e}_{j}} \mathbf{e}_{I}=\mathbf{e}_{j}\right\lrcorner \mathbf{e}_{I} .
$$

Remark 3.6. The $j^{\text {th }}$ right lowering operator in $\mathcal{C} \ell_{p, q}$ is correctly regarded as right contraction according to

$$
\mathbf{e}_{I} D_{j}=\mathbf{e}_{I} \frac{\partial}{\partial \mathbf{e}_{j}}=\mathbf{e}_{I}\left\llcorner\mathbf{e}_{j} .\right.
$$


The raising and lowering operators have a number of interesting properties, including those recalled here. The interested reader is referred to the authors' earlier work for detailed proof and additional properties [18].

The Kronecker delta function appearing below is defined by

$$
\delta_{j k}= \begin{cases}1 & \text { if } j=k, \\ 0 & \text { otherwise. }\end{cases}
$$

Lemma 3.7. Fix nonnegative integers $p, q$ and let $n=p+q$. In $\mathcal{C} \ell_{p, q}$ the operators $\left\{D_{j}: 1 \leq j \leq n\right\}$ and $\left\{R_{j}: 1 \leq j \leq n\right\}$ satisfy the following:

$$
\begin{gathered}
{\left[R_{j}, D_{k}\right]_{+}=\delta_{j k} \mathbf{e}_{j}^{2}, \text { and }} \\
{\left[R_{j}, R_{k}\right]_{+}=\left[D_{j}, D_{k}\right]_{+}=0 .}
\end{gathered}
$$

\section{Weighted raising and lowering operators}

Given a vector $\mathbf{x}=\sum_{i=1}^{n} x_{i} \mathbf{e}_{i}$ in $\mathcal{C} \ell_{p, q}$, the corresponding linear combination of lowering operators has a natural interpretation as a left contraction. In particular, for any $u \in \mathcal{C} \ell_{p, q}$,

$$
\left.\left.\frac{\partial}{\partial \mathbf{x}} u=\left(\bigoplus_{i=1}^{n} x_{i} D_{i}\right) u=\sum_{i=1}^{n} x_{i} \mathbf{e}_{i}\right\lrcorner u=\mathbf{x}\right\lrcorner u .
$$

It also makes sense to define the composition of lowering (differential) operators as multivector left contraction operators. In particular, for canonical grade- $k$ basis blade $\mathbf{e}_{J}$ and arbitrary basis blade $\mathbf{e}_{I}$,

$$
\left.\left.\left.\left.\frac{\partial}{\partial \mathbf{e}_{J_{1}}} \cdots \frac{\partial}{\partial \mathbf{e}_{J_{k}}} \mathbf{e}_{I}=\mathbf{e}_{J_{1}}\right\lrcorner(\cdots\lrcorner\left(\mathbf{e}_{J_{k}}\right\lrcorner \mathbf{e}_{I}\right)\right):=\mathbf{e}_{J}\right\lrcorner \mathbf{e}_{I} .
$$

As a consequence,

$$
\left.\mathbf{e}_{J}\right\lrcorner \mathbf{e}_{I}:= \begin{cases}\vartheta(J, I) \mathbf{e}_{I \backslash J} & \text { if } J \subseteq I, \\ 0 & \text { otherwise. }\end{cases}
$$

This multivector contraction operator extends linearly to all of $\mathcal{C} \ell_{p, q}$. This contraction operator is correctly regarded as a $k^{\text {th }}$ order lowering operator

$$
D_{J} \mathbf{e}_{I}=\vartheta(J, I) \mathbf{e}_{I \backslash J} \in\left\langle\mathcal{C} \ell_{p, q}\right\rangle_{|I|-|J|} .
$$

One defines multivector right contraction operators in similar fashion. 
Due to associativity, the exterior product already has a natural generalization; i.e.,

$$
\mathbf{e}_{I} \wedge \mathbf{e}_{J}=\mathbf{e}_{I_{1}} \wedge \cdots \wedge \mathbf{e}_{I_{|I|}} \wedge \mathbf{e}_{J_{1}} \wedge \cdots \wedge \mathbf{e}_{J_{|J|}} .
$$

Consequently,

$$
\mathbf{e}_{I} \wedge \mathbf{e}_{J}= \begin{cases}\vartheta(I, J) \mathbf{e}_{I \cup J} & \text { if } I \cap J=\emptyset, \\ 0 & \text { otherwise. }\end{cases}
$$

Note that the general blade product $\mathbf{e}_{I} \mathbf{e}_{J}$ has the operator calculus formulation

$$
\mathbf{e}_{I} \mathbf{e}_{J}=\left(\frac{\partial}{\partial \mathbf{e}_{I_{1}}}+R_{I_{1}}\right) \circ \cdots \circ\left(\frac{\partial}{\partial \mathbf{e}_{I|I|}}+R_{I_{|I|}}\right) \mathbf{e}_{J} .
$$

Observe that the linear combination of raising operators in $\mathcal{C} \ell_{p, q}$ also has a natural interpretation as a weighted raising operator of the form

$$
\int u d \mathbf{x}=\left(\bigoplus_{j=1}^{n} x_{j} R_{j}\right) u=\sum_{j=1}^{n} x_{j} \mathbf{e}_{j} \wedge u=\mathbf{x} \wedge u,
$$

and is correctly regarded as a linear operator mapping grade $k$ elements to grade $k+1$ elements for $k=0, \ldots, n-1$.

Property (3.23) of Lemma 3.7 gives the Clifford vector analog of the number operator. The following lemma is obtained as an immediate corollary.

A more general analog to the number operator of quantum mechanics is the signed grade operator defined on blades by

$$
\left.\Gamma\left(\mathbf{u}_{1} \wedge \cdots \wedge \mathbf{u}_{k}\right)=\left(\sum_{\ell=1}^{k} \mathbf{u}_{\ell}\right\lrcorner \mathbf{u}_{\ell}\right) \mathbf{u}_{1} \wedge \cdots \wedge \mathbf{u}_{k} .
$$

Lemma 4.1. In $\mathcal{C} \ell_{p, q}$, the operator sum $\bigoplus_{j=1}^{n}\left(R_{j} D_{j}\right)$ corresponds to the signed grade operator by

$$
\bigoplus_{j=1}^{n} R_{j} D_{j} \mathbf{e}_{I}=\sum_{j \in I} \mathbf{e}_{j}{ }^{2} \mathbf{e}_{I}=\Gamma\left(\mathbf{e}_{I}\right)
$$

Moreover, in the Euclidean Clifford algebra $\mathcal{C} \ell_{n, 0}$ isomorphic to $n$ particle fermion Fock space,

$$
\Gamma\left(\mathbf{e}_{I}\right)=|I| \mathbf{e}_{I} .
$$


Proof. The result follows from Lemma 3.7 by noting that $R_{j} D_{j} \mathbf{e}_{I}=0$ if $j \notin I$.

Since the choice of basis $\left\{\mathbf{e}_{i}: 1 \leq i \leq n\right\}$ for the vector space of generators for $\mathcal{C} \ell_{p, q}$ is arbitrary, it is convenient to pass to the basisfree definition of the Clifford algebra with quadratic form $Q$. With this general framework in mind, the weighted lowering operator is now formally defined. For reasons that will become apparent later, vectors $\mathbf{x}$ used to define the weighted raising and lowering operators are required to be non-null, i.e., it is required that $\mathbf{x}^{2} \neq 0$.

Definition 4.2. Let $\mathrm{x}$ be a non-null vector in $\mathcal{C} \ell(Q)$ and define the weighted lowering operator $\Lambda_{\mathbf{x}}$ on $\mathcal{C} \ell(Q)$ by

$$
\left.\Lambda_{\mathbf{x}} u=\frac{\partial}{\partial \mathbf{x}} u=\mathbf{x}\right\lrcorner u
$$

for any $u \in \mathcal{C} \ell(Q)$.

The weighted (left) lowering operator $\Lambda_{\mathbf{x}}$ is correctly regarded as an operator taking elements of grade $k$ to elements of grade $k-1$ for $k=1, \ldots, n$.

Definition 4.3. Let $\mathrm{x}$ be a non-null vector in $\mathcal{C} \ell(Q)$ and define the corresponding weighted raising operator $\Xi_{\mathbf{x}}$ on $\mathcal{C} \ell(Q)$ by

$$
\Xi_{\mathbf{x}} u=\int u d \mathbf{x}=\mathbf{x} \wedge u
$$

for any $u \in \mathcal{C} \ell(Q)$.

The role of raising and lowering operators in the Clifford (geometric) product is made explicit by considering the left regular representation of multiplication by a vector $\mathbf{x}$. Specifically, this is the operator $\operatorname{sum}\left(\Xi_{\mathbf{x}} \oplus \Lambda_{\mathbf{x}}\right)$, as seen by

$$
\mathbf{x} u=\left(\Xi_{\mathbf{x}} \oplus \Lambda_{\mathbf{x}}\right) u
$$

for $u \in \mathcal{C} \ell(Q)$.

The relationship between the weighted raising and lowering operators is made clear by the next lemma.

Lemma 4.4. For fixed non-null vector $\mathbf{x}$ in $\mathcal{C} \ell(Q)$, the operators $\Xi_{\mathbf{x}}$ and $\Lambda_{\mathbf{x}}$ are dual to each other with respect to the inner product $\langle\cdot, \cdot\rangle_{Q}$; i.e., $\left\langle\Lambda_{\mathbf{x}} u, w\right\rangle_{Q}=\left\langle u, \Xi_{\mathbf{x}} w\right\rangle_{Q}$ for all $u, w \in \mathcal{C} \ell(Q)$.

Proof. The result follows immediately from (3.16) and the definitions of weighted lowering and raising operators. 
Lemma 4.5. For any non-null vector $\mathbf{x} \in \mathcal{C} \ell(Q)$, the corresponding weighted lowering and raising operators are nilpotent of index 2 . That is,

$$
\begin{aligned}
& \Lambda_{\mathrm{x}}^{2}:=\Lambda_{\mathrm{x}} \circ \Lambda_{\mathrm{x}}=0, \\
& \Xi_{\mathrm{x}}^{2}:=\Xi_{\mathrm{x}} \circ \Xi_{\mathrm{x}}=0 .
\end{aligned}
$$

Proof. The result follows from Lemma 3.7 by linearity of lowering and raising operators $D_{j}$ and $R_{j}$.

An immediate consequence is that solutions of the Clifford heat equation must be constant.

Lemma 4.6. For fixed non-null vector $\mathbf{x}$ in $\mathcal{C} \ell(Q)$ and Clifford-valued $u:=u(t)$, the Clifford heat equation

$$
\partial_{t} u=\Lambda_{\mathbf{x}}{ }^{2} u
$$

has only solutions of the form $u(t)=c$ for some constant $c$.

Proof. Index-2 nilpotency of $\Lambda_{\mathbf{x}}$ implies $\partial_{t} u=0$.

\section{$5 \quad$ Clifford Appell systems}

Motivated by polynomial Appell systems, one intuitively expects to define a Clifford Appell system as a pair $\Psi=\left(\left\{\psi_{k}: 1 \leq k \leq n\right\}, D\right)$, where $\left\{\psi_{k}\right\}$ is a collection of Clifford multivectors and $D$ is a lowering operator defined on $\mathcal{C} \ell(Q)$ such that

i. $\psi_{k}$ is a grade- $k$ Clifford multivector for each $k$, and

ii. $D \psi_{k}=\psi_{k-1}$.

A problem with this definition is seen immediately. Assuming $\psi_{k} \neq$ 0 , the index-2 nilpotency of lowering operators seen in Lemma 4.5 gives $\psi_{\ell}=0$ for $0 \leq \ell \leq k-2$ for any fixed lowering operator $D$. In order to define Clifford Appell systems with more than two Clifford multivectors, one must construct a sequence of lowering operators.

Analysis of Clifford Appell systems will be simplified by considering homogeneous Clifford Appell systems defined below. General Clifford Appell systems can then be constructed as sums of homogeneous systems.

Definition 5.1. A homogeneous (left) Clifford Appell system is a pair $\Psi=\left(\left\{\psi_{k}\right\},\left\{\Lambda_{\mathbf{x}_{k}}\right\}\right)$, where $\left\{\psi_{k}\right\} \subset \mathcal{C} \ell(Q)$, and $\left\{\Lambda_{\mathbf{x}_{k}}\right\}$ is a collection of weighted (left) lowering operators associated with a sequence of nonnull Clifford vectors $\left(\mathbf{x}_{k}\right)$ such that

i. for each $k$, either $\psi_{k}=0$ or $\psi_{k}$ is homogeneous of grade $k$, and 
ii. $\Lambda_{\mathbf{x}_{k}} \psi_{k}=\psi_{k-1}$ for each $k$.

One similarly defines a homogeneous right Clifford Appell system in terms of weighted right lowering operators.

Definition 5.2. The order of $\Psi$ is defined as the maximum grade among the elements of $\left\{\psi_{k}\right\}$. The system $\Psi$ is said to be of rank $r$ if $\left\{\psi_{k}\right\}$ contains $r+1$ nonzero elements .

This definition of rank follows naturally from Appell systems of polynomials of a single variable, since a polynomial's degree is always one greater than its number of nonzero derivatives.

Remark 5.3. In $\mathcal{C} \ell_{n, 0}$, which is canonically isomorphic to the $n$-particle fermionic Fock space, the lowering operator maps monomials representing $k$-particle systems to monomials representing $(k-1)$-particle systems. In other words, the lowering operator acts as an annihilation operator.

Remark 5.4. Fixing nonzero $\psi_{n}$ and $\mathbf{x}_{k}=\mathbf{x} \in V$ for each $k$ results in a rank-1 homogeneous Clifford Appell system by the index-2 nilpotent property of lowering operators .

Lemma 5.5. Let $X=\left\{\mathbf{x}_{k}: 1 \leq k \leq m\right\}$ be a collection of vectors in the $n$-dimensional vector space $V$. Then, the exterior product

$$
\mathbf{x}_{1} \wedge \cdots \wedge \mathbf{x}_{m}
$$

is nonzero in $\mathcal{C} \ell(Q)$ if and only if $X$ is linearly independent.

Proof. When the set $X$ is linearly independent, the exterior product represents an oriented $m$-dimensional volume element, or pseudoscalar, in the subspace of $V$ spanned by $X$.

On the other hand, if $X$ is not linearly independent, let $1 \leq \ell \leq m$ and suppose $\mathbf{x}_{\ell}=\sum_{\substack{1 \leq k, \ell, \leq m \\ k \neq \ell}} \alpha_{k} \mathbf{x}_{k}$ for scalars $\left\{\alpha_{k}\right\}$. Then, relabeling vectors in the set complement $\left\{\mathbf{x}_{1}, \ldots, \mathbf{x}_{m}\right\} \backslash \mathbf{x}_{\ell}=\left\{\mathbf{y}_{1}, \ldots, \mathbf{y}_{m-1}\right\}$, one finds (with sign changes as appropriate)

$$
\begin{aligned}
& \mathbf{x}_{1} \wedge \cdots \wedge \mathbf{x}_{m}= \pm \mathbf{x}_{\ell} \wedge \mathbf{y}_{1} \wedge \cdots \wedge \mathbf{y}_{m-1} \\
&= \pm\left(\sum_{j=1}^{m-1} \alpha_{j} \mathbf{y}_{j}\right) \wedge \mathbf{y}_{1} \wedge \cdots \wedge \mathbf{y}_{m-1} \\
&= \pm \mathbf{y}_{2} \wedge \cdots \wedge\left(\mathbf{y}_{1} \wedge \mathbf{y}_{1}\right)+\sum_{j=2}^{m-1} \pm \mathbf{y}_{1} \wedge \cdots \wedge\left(\mathbf{y}_{j} \wedge \mathbf{y}_{j}\right)
\end{aligned}
$$

Whence, index-2 nilpotency of the wedge product (i.e., raising operators) makes all terms zero. 
Writing the vectors $\left\{\mathbf{x}_{k}\right\}$ with respect to the orthonormal basis $\left\{\mathbf{e}_{i}\right.$ : $1 \leq i \leq n\}$ for $V$, the exterior product can be expressed in the following way: the coefficient of the $m$-vector $\mathbf{e}_{I}$ for $I=\left\{I_{1}, \ldots, I_{m}\right\} \subseteq[n]$ in the canonical expansion of the wedge product is given by the determinant of the submatrix

$$
\operatorname{det}_{I}\left(\begin{array}{c}
\mathbf{x}_{1} \\
\vdots \\
\mathbf{x}_{m}
\end{array}\right)=\operatorname{det}\left(\begin{array}{cccc}
x_{1, I_{1}} & x_{1, I_{2}} & \ldots & x_{1, I_{m}} \\
x_{2, I_{1}} & x_{2, I_{2}} & \ldots & x_{2, I_{m}} \\
\ldots & \ldots & \ldots & \ldots \\
x_{m, I_{1}} & x_{m, I_{2}} & \ldots & x_{m, I_{m}}
\end{array}\right)
$$

This determinant is the scalar coefficient of the pseudoscalar in the projection of $\mathbf{x}_{1} \wedge \cdots \wedge \mathbf{x}_{m}$ onto the $m$-dimensional subspace of $V$ spanned by $\left\{\mathbf{e}_{I_{1}}, \ldots, \mathbf{e}_{I_{m}}\right\}$. It follows that all such projections are zero if and only if $X$ is linearly dependent.

Remark 5.6. In terms of the Clifford (geometric) product, the element $\mathbf{x}_{1} \cdots \mathbf{x}_{m}$ is an $m$-blade in $\mathcal{C} \ell(Q)$ if and only if the set $\left\{\mathbf{x}_{1}, \ldots, \mathbf{x}_{m}\right\}$ is orthogonal with respect to the $Q$-inner product.

To ensure $\psi_{k} \neq 0$ for all $1 \leq k \leq n$, appropriate conditions on the sequence $\left(\mathbf{x}_{k}\right)$ are addressed in the next proposition. Note that in any homogeneous Clifford Appell system associated with an $n$-dimensional vector space $V, \psi_{n}$ is associated with a scalar multiple of the pseudoscalar $\psi_{n}=\alpha \mathbf{e}_{[n]}$.

Lemma 5.7. Let $\left\{B_{i}: 1 \leq i \leq M \leq\left(\begin{array}{l}n \\ k\end{array}\right)\right\}$ be a collection of distinct $k$-blades in $\mathcal{C} \ell(Q)$, and let $\mathbf{x}$ be a non-null Clifford vector. Then, $\mathbf{x}\lrcorner\left(\sum a_{i} B_{i}\right)=0$ if and only if $\left.\mathbf{x}\right\lrcorner\left(a_{i} B_{i}\right)=0$ for each $i$.

Proof. One direction of the proof is obvious. On the other hand, suppose that for some subset $S$ of indices, terms $\left.\{\mathbf{x}\lrcorner\left(a_{i} B_{i}\right): i \in S\right\}$ are nonzero. Rewrite $\sum_{i \in S} a_{i} B_{i}$ in terms of a new orthonormal basis of the form $X=\left\{\mathbf{x}, \mathbf{f}_{1}, \ldots, \mathbf{f}_{n-1}\right\}$ obtained from the canonical basis by Gram-Schmidt orthogonalization with respect to $\langle\cdot, \cdot\rangle_{Q}$. Letting $I_{i}$ denote the subset of the basis $X$ associated with the canonical blade $B_{i}$ and letting $b_{i}$ denote appropriate nonzero coefficients,

$$
\begin{aligned}
\left.\mathbf{x}\lrcorner\left(\sum_{i \in S} a_{i} B_{i}\right)=\sum_{i \in S} a_{i}(\mathbf{x}\lrcorner\left(b_{i} \mathbf{x} \wedge \bigwedge_{j \in I_{i}} \mathbf{f}_{j}\right)\right) & \\
& =\sum_{i \in S}\left(a_{i} b_{i} \bigwedge_{j \in I_{i}} \mathbf{f}_{j}\right)=\sum_{i \in S} a_{i} b_{i} \mathbf{f}_{I_{i}},
\end{aligned}
$$

which is nonzero by linear independence of the $(k-1)$-blades $\left\{\mathbf{f}_{I_{i}}\right\}$. 
Proposition 5.8. Let $\Psi=\left(\left\{\psi_{k}\right\},\left\{\Lambda_{\mathbf{x}_{k}}\right\}\right)$ be an order-n homogeneous Clifford Appell system. The rank of $\Psi$ is $m$ if and only if the set $X=\left\{\mathbf{x}_{n-m+1}, \ldots, \mathbf{x}_{n}\right\}$ is linearly-independent; i.e., $\operatorname{rank}(X)=m$.

Proof. By definition,

$$
\begin{aligned}
& \psi_{n-m}=\frac{\partial}{\partial \mathbf{x}_{n-m+1}}\left(\cdots\left(\frac{\partial}{\partial \mathbf{x}_{n}} \psi_{n}\right)\right) \\
&\left.\left.\left.\left.=\mathbf{x}_{n-m+1}\right\lrcorner\left(\mathbf{x}_{n-m+2}\right\lrcorner(\cdots\lrcorner\left(\mathbf{x}_{n}\right\lrcorner \psi_{n}\right)\right)\right) \\
& \quad=\left(\mathbf{x}_{n-m+1} \wedge \cdots \wedge \mathbf{x}_{n}\right\lrcorner \psi_{n} .
\end{aligned}
$$

Observing that $\psi_{n}$ is a scalar multiple of the pseudoscalar, it follows that $\left.\mathbf{x}_{k}\right\lrcorner \psi_{n}$ is homogeneous of grade $n-1$ for each $k$. Moreover, it follows that $\psi_{n-m} \neq 0$ if and only if $\psi_{n-m}$ is homogeneous of grade $n-m$; i.e., $\left(\mathbf{x}_{n-m+1} \wedge \cdots \wedge \mathbf{x}_{n}\right)$ must be an $m$-blade.

Note that Proposition 5.8 guarantees the existence of an order- $n$, rank- $n$ homogeneous Clifford Appell system associated with any basis of the vector space $V$ generating $\mathcal{C} \ell(Q)$. The construction algorithm is straightforward:

1. Order the basis $\left\{\mathbf{v}_{1}, \ldots, \mathbf{v}_{n}\right\}$ for $V$.

2. Set $\psi_{n}=\alpha \mathbf{e}_{[n]}$ for some scalar $\alpha$.

3. Set $\left.\psi_{k-1}=\mathbf{v}_{k}\right\lrcorner \psi_{k}$ for each $k=n, n-1, \ldots, 1$.

Further, note that Proposition 5.8 does not guarantee the existence of such a system in infinite-dimensional Clifford algebras. Moreover, it does not address the construction of invertible Appell systems as defined below.

Definition 5.9. An invertible homogeneous (left) Clifford Appell system is a pair $\Psi=\left(\left\{\psi_{k}\right\},\left\{\Lambda_{\mathbf{x}_{k}}\right\}\right)$, where $\left\{\psi_{k}\right\} \subset \mathcal{C} \ell(Q)$, and $\left\{\Lambda_{\mathbf{x}_{k}}\right\}$ is a collection of weighted (left) lowering operators such that

i. for each $k, \psi_{k}$ is homogeneous of grade $k$, and

ii. $\Lambda_{\mathbf{x}_{k}} \psi_{k}=\psi_{k-1}$ for each $k$, and

iii. $\Xi_{\mathbf{x}_{k}} \psi_{k-1}=\psi_{k}$ for each $k$.

Theorem 5.10 (Solutions). Let $\mathbf{x}$ be a non-null Clifford vector, and let $\psi_{k-1}$ be a grade- $(k-1)$ Clifford multivector. Then, the Clifford equation

$$
\frac{\partial}{\partial \mathbf{x}} \psi_{k}=\psi_{k-1}
$$

has a solution $\psi_{k}$ if and only if $\frac{\partial}{\partial \mathbf{x}} \psi_{k-1}=0$. Moreover, a solution of (5.5) is the homogeneous grade- $k$ multivector given by $\psi_{k}=$ $\frac{1}{\mathbf{x}^{2}} \int \psi_{k-1} d \mathbf{x}$. 
Proof. Suppose $\frac{\partial}{\partial \mathbf{x}} \psi_{k}=\psi_{k-1}$ holds for some grade- $k$ element $\psi_{k}$. Index-2 nilpotency of lowering operators then gives

$$
\frac{\partial}{\partial \mathbf{x}} \psi_{k-1}=\frac{\partial}{\partial \mathbf{x}} \frac{\partial}{\partial \mathbf{x}} \psi_{k}=0
$$

On the other hand, if $\frac{\partial}{\partial \mathbf{x}} \psi_{k-1}=0$, then $\mathbf{x} \wedge \psi_{k-1}=\mathbf{x} \psi_{k-1}$ since $\mathbf{x}$ must be orthogonal to any blades appearing in the expansion of $\psi$ by Lemma 5.7. Moreover, $\mathbf{x}$ orthogonal to $u_{1}, \ldots, u_{k}$ implies $\left.\mathbf{x}\right\lrcorner\left(\mathbf{x} \wedge u_{1} \wedge\right.$ $\left.\cdots \wedge u_{k}\right)=\mathbf{x}^{2}\left(u_{1} \wedge \cdots \wedge u_{k}\right)$. Whence letting $\psi_{k}=\mathbf{x} \wedge \psi_{k-1}$ gives

$$
\left.\frac{\partial}{\partial \mathbf{x}} \psi_{k}=\frac{\partial}{\partial \mathbf{x}}\left(\mathbf{x} \wedge \psi_{k-1}\right)=\mathbf{x}\right\lrcorner\left(\mathbf{x} \psi_{k-1}\right)=\mathbf{x} \mathbf{x} \psi_{k-1}=\mathbf{x}^{2} \psi_{k-1} .
$$

In light of Theorem 5.10, fixing $\mathbf{x}_{k} \in V$ and considering

$$
\Lambda_{\mathbf{x}_{k}}:\langle\mathcal{C} \ell(Q)\rangle_{k-1} \cup\langle\mathcal{C} \ell(Q)\rangle_{k} \rightarrow\langle\mathcal{C} \ell(Q)\rangle_{k-2} \cup\langle\mathcal{C} \ell(Q)\rangle_{k-1},
$$

define the grade- $k$ subspace

$$
\mathcal{A}_{k}=\Xi_{\mathbf{x}_{k}}\left(\left\langle\operatorname{ker} \Lambda_{\mathbf{x}_{k}}\right\rangle_{k}\right) \subset\langle\mathcal{C} \ell(Q)\rangle_{k} .
$$

Then, letting $\mathcal{I}$ denote the identity operator, one finds

$$
\left.\Xi_{\mathbf{x}_{k}} \Lambda_{\mathbf{x}_{k}}\right|_{\mathcal{A}_{k}}=\mathbf{x}_{k}^{2} \mathcal{I}
$$

Corollary 5.11. [Appell System Solutions] Let $\left\{\mathbf{x}_{k}: 1 \leq k \leq m\right\}$ be an ordered collection of vectors, orthogonal with respect to the quadratic form $Q$. Let $\psi_{0}$ be a scalar. Then, setting

$$
\psi_{k}:=\frac{1}{\mathbf{x}_{k}^{2}} \int \psi_{k-1} d \mathbf{x}_{k}
$$

for each $k=1, \ldots, m$ gives an order- $m$, rank-m invertible homogeneous Clifford Appell system $\Psi=\left(\left\{\psi_{k}\right\},\left\{\Lambda_{\mathbf{x}_{k}}\right\}\right)$.

\subsection{Heterogeneous Clifford Appell systems}

A more general definition of Clifford Appell systems is now developed by considering heterogeneous multivectors. By extending results for solutions of homogenous systems, it is possible to construct invertible heterogeneous Clifford Appell systems. 


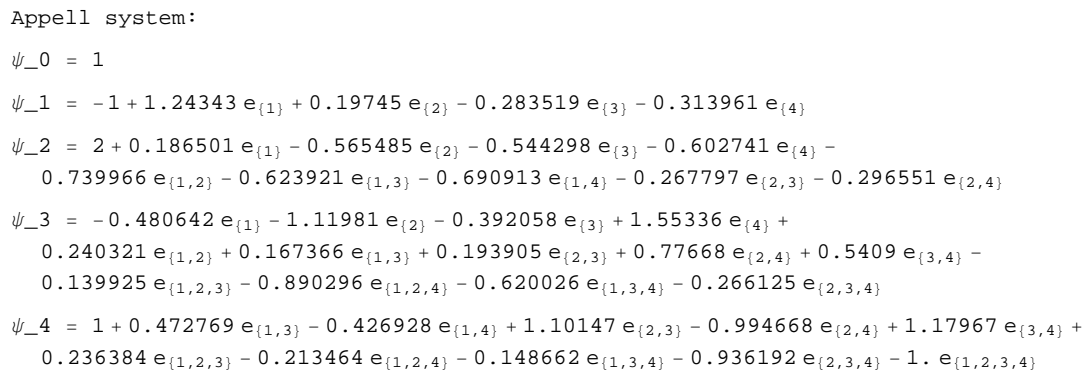

Figure 1: Heterogeneous Appell multivectors in $\mathcal{C} \ell_{1,3}$ associated with sequence $\left(\mathbf{x}_{k}\right)$ of Example 5.15.

Definition 5.12. A heterogeneous (left) Clifford Appell system is a pair

$$
\Psi=\left(\left\{\psi_{k}\right\},\left\{\Lambda_{\mathbf{x}_{k}}\right\}\right),
$$

where $\left\{\psi_{k}: 1 \leq k \leq n\right\}$ is a collection of Clifford multivectors, and $\left\{\Lambda_{\mathbf{x}_{k}}: 1 \leq k \leq n\right\}$ is a collection of weighted (left) lowering operators such that

i. $\psi_{k}$ is a heterogeneous, grade- $k$ Clifford multivector for each $k$ and

ii. $\Lambda_{\mathbf{x}_{k}} \psi_{k}=\psi_{k-1}$ for each $k$.

Theorem 5.13. Let $\left\{\mathbf{x}_{k}: 1 \leq k \leq m\right\}$ be an ordered collection of vectors, orthogonal with respect to the quadratic form $Q$. Let $\left\{c_{1}, \ldots, c_{m}\right\}$ be a collection of scalars, at least one of which is nonzero, and set $\psi_{0}:=c_{0} \neq 0$. Then, setting

$$
\psi_{k}:=\frac{1}{\mathbf{x}_{k}^{2}} \int \psi_{k-1} d \mathbf{x}_{k}+c_{k}
$$

for each $k=1, \ldots, m$ gives an order- $m$, rank- $m$ heterogeneous Clifford Appell system $\Psi=\left(\left\{\psi_{k}\right\},\left\{\Lambda_{\mathbf{x}_{k}}\right\}\right)$.

Proof. By induction on $1 \leq \ell \leq m$, it will be shown that $\psi_{\ell}$ is of the form

$$
\begin{array}{r}
\psi_{\ell}=\frac{c_{0}}{\prod_{j=1}^{\ell} \mathbf{x}_{j}^{2}}\left(\mathbf{x}_{\ell} \wedge \cdots \wedge \mathbf{x}_{1}\right)+\frac{c_{1}}{\prod_{j=1}^{\ell} \mathbf{x}_{j}^{2}}\left(\mathbf{x}_{\ell} \wedge \cdots \wedge \mathbf{x}_{2}\right)+ \\
+\frac{c_{2}}{\prod_{j=3}^{\ell} \mathbf{x}_{j}^{2}}\left(\mathbf{x}_{\ell} \wedge \cdots \wedge \mathbf{x}_{3}\right)+\cdots+\frac{c_{\ell-1}}{\mathbf{x}_{\ell}{ }^{2}} \mathbf{x}_{\ell}+c_{\ell}
\end{array}
$$

When $\ell=1$, setting

$$
\psi_{1}:=\frac{c_{0}}{\mathbf{x}_{1}{ }^{2}} \int \psi_{0} d \mathbf{x}_{1}+c_{1}=\frac{c_{0}}{\mathbf{x}_{1}{ }^{2}} \mathbf{x}_{1}+c_{1},
$$


which establishes the basis step of the induction. Assuming $\psi_{\ell}$ is of the form (5.13), it follows that

$$
\begin{aligned}
& \psi_{\ell+1}=\frac{1}{\mathbf{x}_{\ell+1}{ }^{2}} \int \psi_{\ell} d \mathbf{x}_{\ell+1}+c_{\ell}=\frac{1}{\mathbf{x}_{\ell+1}{ }^{2}} \frac{c_{0}}{\prod_{j=1}^{\ell} \mathbf{x}_{j}^{2}} \mathbf{x}_{\ell+1} \wedge\left(\mathbf{x}_{\ell} \wedge \cdots \wedge \mathbf{x}_{1}\right) \\
&+\frac{1}{\mathbf{x}_{\ell+1}{ }^{2}} \frac{c_{1}}{\prod_{j=1}^{\ell} \mathbf{x}_{j}^{2}} \mathbf{x}_{\ell+1} \wedge\left(\mathbf{x}_{\ell} \wedge \cdots \wedge \mathbf{x}_{2}\right)+ \\
&+\frac{1}{\mathbf{x}_{\ell+1}{ }^{2}} \frac{c_{2}}{\prod_{j=3}^{\ell} \mathbf{x}_{j}^{2}} \mathbf{x}_{\ell} \wedge\left(\mathbf{x}_{\ell} \wedge \cdots \wedge \mathbf{x}_{3}\right)+\cdots \\
& \cdots+\frac{1}{\mathbf{x}_{\ell+1}{ }^{2}} \frac{c_{\ell-1}}{\mathbf{x}_{\ell}{ }^{2}} \mathbf{x}_{\ell+1} \wedge \mathbf{x}_{\ell}+\frac{1}{\mathbf{x}_{\ell+1}{ }^{2}} c_{\ell} \mathbf{x}_{\ell+1}+c_{\ell+1} .
\end{aligned}
$$

Simplifying completes the inductive step.

To see that the system is a rank- $m$ Clifford Appell system, consider the action of $\Lambda_{\mathbf{x}_{\ell}}$ on $\psi_{\ell}$ for $1 \leq \ell \leq m$. Since the vectors $\left\{\mathbf{x}_{\ell}\right\}$ are $Q$ orthogonal and non-null, one immediately finds

$$
\begin{gathered}
\left.\left.\Lambda_{\mathbf{x}_{\ell}} \psi_{\ell}=\mathbf{x}_{\ell}\right\lrcorner \psi_{\ell}=\frac{c_{0}}{\prod_{j=1}^{\ell} \mathbf{x}_{j}^{2}} \mathbf{x}_{\ell}\right\lrcorner\left(\mathbf{x}_{\ell} \wedge \cdots \wedge \mathbf{x}_{1}\right) \\
\left.\left.+\frac{c_{1}}{\prod_{j=1}^{\ell} \mathbf{x}_{j}^{2}} \mathbf{x}_{\ell}\right\lrcorner\left(\mathbf{x}_{\ell} \wedge \cdots \wedge \mathbf{x}_{2}\right)+\frac{c_{2}}{\prod_{j=3}^{\ell} \mathbf{x}_{j}^{2}} \mathbf{x}_{\ell}\right\lrcorner\left(\mathbf{x}_{\ell} \wedge \cdots \wedge \mathbf{x}_{3}\right) \\
\left.\left.\quad+\cdots+\mathbf{x}_{\ell}\right\lrcorner \frac{c_{\ell-1}}{\mathbf{x}_{\ell}{ }^{2}} \mathbf{x}_{\ell}+\mathbf{x}_{\ell}\right\lrcorner c_{\ell} \\
=\frac{c_{0}}{\prod_{j=1}^{\ell-1} \mathbf{x}_{j}^{2}}\left(\mathbf{x}_{\ell-1} \wedge \cdots \wedge \mathbf{x}_{1}\right)+\frac{c_{1}}{\prod_{j=1}^{\ell-1} \mathbf{x}_{j}^{2}}\left(\mathbf{x}_{\ell-1} \wedge \cdots \wedge \mathbf{x}_{2}\right)+ \\
+\frac{c_{2}}{\prod_{j=3}^{\ell-1} \mathbf{x}_{j}{ }^{2}}\left(\mathbf{x}_{\ell-1} \wedge \cdots \wedge \mathbf{x}_{3}\right)+\cdots+\frac{c_{\ell-1}}{\mathbf{x}_{\ell-1}{ }^{2}} \mathbf{x}_{\ell-1}+c_{\ell-1} \\
=\psi_{\ell-1} .
\end{gathered}
$$

Remark 5.14. Note that if $c_{0}$ is the only nonzero scalar in the collection $\left\{c_{0}, \ldots, c_{m}\right\}$, the system $\Psi$ is homogeneous.

Example 5.15. Let $\left(c_{0}, \ldots, c_{4}\right):=(1,-1,2,0,1)$, and consider the $Q$-orthogonal Mathematica-generated vector sequence

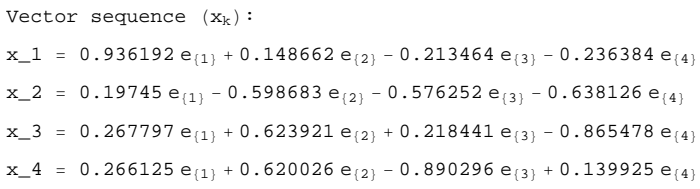

in $\mathcal{C} \ell_{1,3}$. The heterogeneous Clifford Appell system of Figure 1 was obtained by defining $\psi_{0}:=c_{0}$ and proceeding inductively by setting $\psi_{k}:=\Xi_{\mathbf{x}_{k}} \psi_{k-1}+c_{k}$ for $k=1, \ldots, n$. 


\subsection{Role of blade factorization in the construction of Appell systems}

A brief discussion of blade factorization algorithms is included here because they have a natural connection to solutions of invertible Appell systems in Clifford algebras. Examples of blade factorization algorithms can be found in works by Dorst, Fontijne, et al. [5], [10].

Definition 5.16. A blade factorization algorithm is defined as any mapping $\neg: \mathcal{C} \ell(Q) \rightarrow \bigoplus_{k=1}^{n}\left(\langle\mathcal{C} \ell(Q)\rangle_{1}\right)^{k}$ such that for any $m$-blade $u \in$ $\mathcal{C} \ell(Q):$

1. $\left.\urcorner(u)=\left(\neg(u)_{1}, \ldots,\right\urcorner(u)_{m}\right)$ is an ordered $m$-tuple of non-null, $Q$-orthogonal Clifford vectors (1-blades), and

2. $\left.u=\alpha\urcorner(u)_{1} \cdots\right\urcorner(u)_{m}$ for some nonzero scalar $\alpha$.

In light of Theorem 5.10 and Corollary 5.11, any $m$-blade $\psi_{m}$ can serve as the top-term of an order- $m$, rank- $m$ invertible homogeneous Clifford Appell system $\left(\left\{\psi_{k}\right\}, \Lambda_{\mathbf{x}_{k}}\right)$, given a $Q$-orthogonal collection $\left\{\mathbf{x}_{k}\right\}$ such that

$$
\psi_{m}=\mathbf{x}_{1} \wedge \cdots \wedge \mathbf{x}_{k} .
$$

The constituent vectors $\mathbf{x}_{k}$ of $\psi_{m}$ can be obtained by applying a blade factorization algorithm to the $m$-blade $\psi_{m}$. The factorization recovered, however, is not uniquely determined. Any blade factorization algorithm acting on $\psi_{m}$ determines such an Appell system.

Theorem 5.17. Let $\psi_{m}$ be any $m$-blade in $\mathcal{C} \ell(Q)$. Then, for any blade factorization algorithm $\urcorner$, the $m$-tuple $\rceil\left(\psi_{m}\right)$ determines an order-m, rank-m invertible homogeneous Clifford Appell system by

$$
\left.\left.\psi_{\ell-1}:=\right\urcorner\left(\psi_{m}\right)_{\ell}\right\lrcorner \psi_{\ell}
$$

for $\ell=1, \ldots, k$.

Proof. The result follows immediately from definitions.

\section{Fermion algebras and the fermion field}

Of particular interest in the field of quantum probability, the Clifford algebra $\mathcal{C} \ell_{n, n}$ is known to be isomorphic to the $n$-particle fermion algebra $\mathcal{F}_{n}$. In fact, stochastic processes on Clifford algebras have historically been studied primarily in the form of processes in the fermion field (cf. [1], [2], [3]). Other examples include the central limit theorems developed by von Waldenfels [23] and Hudson [11]. 
Denoting the $j^{\text {th }}$ creation operator by $f_{j}{ }^{+}$and the $j^{\text {th }}$ annihilation operator by $f_{j}$, the isomorphism $\mathcal{F}_{n} \rightarrow \mathcal{C} \ell_{n, n}$ is made explicit by

$$
\begin{gathered}
f_{j}^{+} \mapsto \frac{1}{2}\left(\mathbf{e}_{j}+\mathbf{e}_{n+j}\right), \\
f_{j} \mapsto \frac{1}{2}\left(\mathbf{e}_{j}-\mathbf{e}_{n+j}\right) .
\end{gathered}
$$

Regarding $f_{j}^{+}$and $f_{j}$ as raising and lowering operators on $\mathcal{C} \ell_{n, n}$, respectively, one again finds the left regular representation of multiplication by $\mathbf{e}_{j}$ given as an operator sum

$$
\left(f_{j}^{+}+f_{j}\right) u=\mathbf{e}_{j} u
$$

for arbitrary $u \in \mathcal{C} \ell_{n, n}$.

Example 6.1. Consider the Mathematica-generated collection of orthogonal non-null vectors

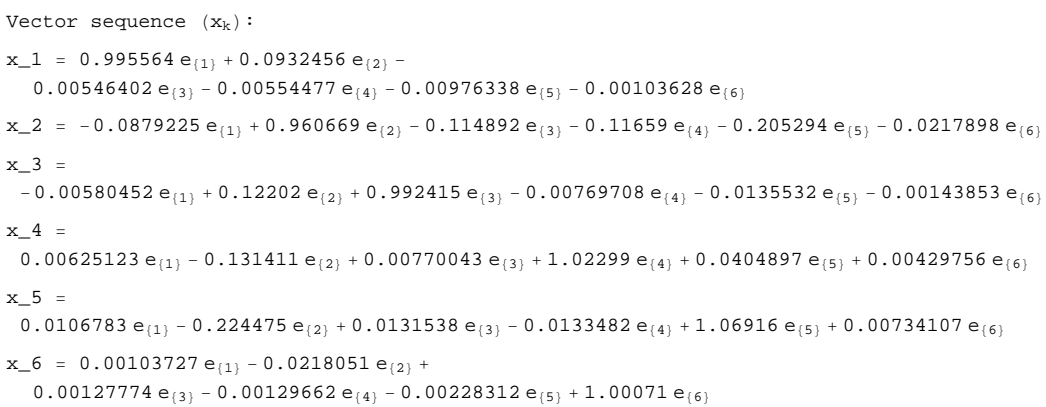

in $\mathcal{C} \ell_{3,3}$, which is canonically isomorphic to the 3 -particle fermion algebra $\mathcal{F}_{3}$. By defining $\psi_{0}:=1$ and proceeding inductively by setting $\psi_{k}:=\Xi_{\mathbf{x}_{k}} \psi_{k-1}$ for $k=1, \ldots, n$, the invertible homogeneous Clifford Appell system of Figure 2 was obtained.

The infinite-dimensional extension is the fermion field,

$$
\mathcal{F} \cong \bigoplus_{n=1}^{\infty} \mathcal{C} \ell_{n, n} .
$$

Infinite-dimensional Clifford algebras (of arbitrary signature) can be easily defined using vectors in a separable Hilbert space $\mathcal{H}$ equipped with a quadratic form $Q$. Generally speaking, for any orthonormal basis $\left\{\mathbf{e}_{i}: 1 \leq i\right\}$ of $\mathcal{H}$ and $\mathbf{x} \in \mathcal{H}$ satisfying $\|\mathbf{x}\|<\infty$, the quadratic form $Q$ acts according to

$$
Q(\mathbf{x})=\sum_{i} \gamma_{i}\left\langle\mathbf{x}, \mathbf{e}_{i}\right\rangle^{2},
$$




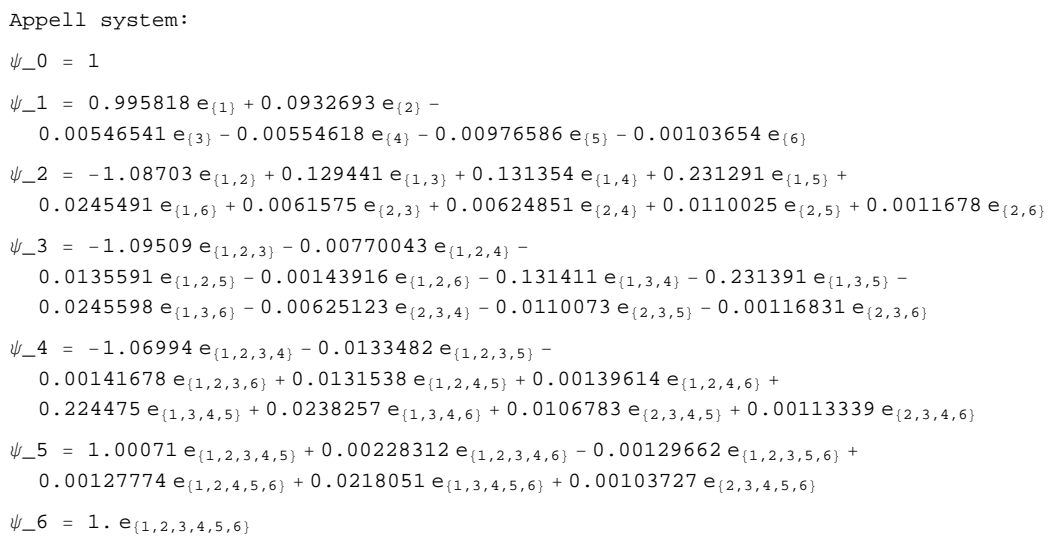

Figure 2: Invertible homogeneous Appell multivectors in $\mathcal{C} \ell_{3,3}$ associated with sequence $\left(\mathbf{x}_{k}\right)$ of Example 6.1.

where $\gamma_{i}= \pm 1$ for each $i$. With this quadratic form in hand, the symmetric bilinear form $\langle\cdot, \cdot\rangle_{Q}$ is defined as in the finite case by

$$
\langle\mathbf{x}, \mathbf{y}\rangle_{Q}=\frac{1}{2}[Q(\mathbf{x}+\mathbf{y})-Q(\mathbf{x})-Q(\mathbf{y})]
$$

for $\mathbf{x}, \mathbf{y} \in \mathcal{H}$.

Left- and right-contraction are again defined in terms of the quadratic form $Q$ as in (3.13), and the Clifford integrals are defined as in (3.2).

In the infinite-dimensional case, one defines the weighted raising and lowering operators by

$$
\begin{aligned}
& \Xi_{\mathbf{x}}=\sum_{j} x_{j} R_{j} \\
& \Lambda_{\mathbf{x}}=\sum_{j} x_{j} D_{j}
\end{aligned}
$$

where $\langle\mathbf{x}, \mathbf{x}\rangle=\sum_{j} x_{j}^{2}=1$.

Considering the quadratic form $Q$ defined on separable Hilbert space $\mathcal{H}$ having countable orthonormal basis $\mathcal{B}=\left\{\mathbf{e}_{i}\right\}$ by

$$
Q(\mathbf{x})=\sum_{i}\left\langle\mathbf{x}, \mathbf{e}_{2 i}\right\rangle^{2}-\sum_{i}\left\langle\mathbf{x}, \mathbf{e}_{2 i-1}\right\rangle^{2}
$$

one obtains an infinite-dimensional Clifford algebra $\mathcal{C} \ell(Q)$ isomorphic 
to the fermion field via the correspondence

$$
\begin{aligned}
f_{j}^{+} & \mapsto \frac{1}{2}\left(\mathbf{e}_{2 j}+\mathbf{e}_{2 j-1}\right), \\
f_{j} & \mapsto \frac{1}{2}\left(\mathbf{e}_{2 j}-\mathbf{e}_{2 j-1}\right) .
\end{aligned}
$$

\subsection{Further remarks on evolution equations}

Returning to solutions of evolution equations, it now becomes apparent that for interesting (i.e., non-constant) discrete processes on Clifford algebras, the "correct" forms of evolution equations (2.10), (2.11), and (2.12) are respectively

$$
\begin{gathered}
\partial_{t} u(k)=\Lambda_{\mathbf{x}_{k}} u(k), \\
\partial_{t} u(k)=\Xi_{\mathbf{x}_{k}} u(k), \text { and } \\
\partial_{t} u(k)=\left(\Lambda_{\mathbf{x}_{k}}+\Xi_{\mathbf{x}_{k}}\right) u(k) .
\end{gathered}
$$

That is, the differential $\Delta u$ at the $k^{\text {th }}$ time step

$$
\partial_{t} u(k):=u(k)-u(k-1)
$$

in the discrete process is given by the action of weighted raising and lowering operators associated with a vector-valued process $\left(\mathbf{x}_{k}\right)$ in the vector space $V$ generating $\mathcal{C} \ell(Q)$. The Clifford-valued process then becomes an additive process of the general form

$$
u(k):=\sum_{\ell=0}^{k} \psi_{j \ell},
$$

where $\left(j_{\ell}\right)$ is a sequence of Appell indices determined by the choice of raising, lowering, or raising+lowering.

The current authors have considered both time-homogeneous discrete processes (cf. [16]) and dynamic processes (cf. [17]) associated with evolution equation (6.14) by defining multiplicative walks on basis blades considering the induced additive walks in Clifford algebras of arbitrary signature.

Example 6.2. Letting $\left(\mathbf{x}_{k}: 1 \leq k\right)$ denote a random sequence of basis vectors from $\mathcal{B}$, consider the evolution equation

$$
\partial_{t} u=\left(\Lambda_{\mathbf{x}_{k}}+\Xi_{\mathbf{x}_{k}}\right) u .
$$

This equation is associated with a discrete additive process $(u(k))$ in $\mathcal{F}$ associated with a random walk on an infinite-dimensional directed hypercube. In particular, consecutive increments of the process are 
associated with adjacent vertices in the hypercube. The process $(u(k))$ in $\mathcal{C} \ell(Q)$ is of the form

$$
u(k)=u(k-1)+\left(\Lambda_{\mathbf{x}_{k}}+\Xi_{\mathbf{x}_{k}}\right) u(k-1) .
$$

Setting $\Upsilon_{\ell}:=\Lambda_{\mathbf{x}_{\ell}}+\Xi_{\mathbf{x}_{\ell}}$ for each $\ell$, and using back-substitution, $u(k+1)$ is given by the ordered product

$$
u(k)=\left(\mathcal{I}+\Upsilon_{k}\right)\left(\mathcal{I}+\Upsilon_{k-1}\right) \cdots\left(\mathcal{I}+\Upsilon_{1}\right) u_{0},
$$

where $u_{0}=u(0)$ is the initial value of the process. Expanding and rewriting with ordered products of lowering and raising operators, one finds

$$
u(k)=\sum_{g=0}^{k} \sum_{\substack{\left(j_{1}, \ldots, j g\right) \in[k] \\ j_{1}>\cdots>j_{g}}}\left(\Lambda_{\mathbf{x}_{j_{1}}}+\Xi_{\mathbf{x}_{j_{1}}}\right) \cdots\left(\Lambda_{\mathbf{x}_{j_{g}}}+\Xi_{\mathbf{x}_{j_{g}}}\right) u_{0} .
$$

Implicitly defining the index function $\nu: \mathcal{B} \rightarrow \mathbb{N}$ by $\mathbf{x}=\mathbf{e}_{\nu(\mathbf{x})}$, the equivalent process in $\mathcal{F}$ is expressed by setting $u_{0}=\mathbf{1}$ to represent the vacuum state and expanding, using the canonical isomorphism $\mathcal{F} \simeq$ $\mathcal{C} \ell(Q)$. One thereby obtains the following general expression for the $k^{\text {th }}$ value of the process:

$$
u(k)=\sum_{g=0}^{k} \sum_{\substack{\left(j_{1} \ldots, j_{g}\right) \in[k] g \\ j_{1}>\cdots, j_{g}}}\left(f_{\nu\left(\mathbf{x}_{j_{1}}\right)}+f_{\nu\left(\mathbf{x}_{j_{1}}\right)}+\right) \cdots\left(f_{\nu\left(\mathbf{x}_{j_{g}}\right)}+f_{\nu\left(\mathbf{x}_{j_{g}}\right)}\right) \mathbf{1} .
$$

\section{Conclusion}

The authors have shown methods for constructing invertible Appell systems in Clifford algebras of arbitrary signature. As seen in Examples 5.15 and 6.1, these methods readily lend themselves to convenient symbolic computations and can be used for further research involving Clifford evolution equations.

\section{References}

[1] D. Applebaum, Fermion stochastic calculus in Dirac-Fock space, $J$. Phys. A, 28 257-270, (1995).

[2] D. Applebaum, R. Hudson, Fermion Itô's formula and stochastic evolutions, Comm. Math. Phys., 96 473-496, (1984).

[3] C. Barnett, R. Streater, I. Wilde, The Itô-Clifford integral I, J. Functional Analysis, 48 172-212, (1982). 
[4] D. Aerts, M. Czachor, Cartoon computation: Quantum-like algorithms without quantum mechanics, J. Phys. A: Math. Theor. 40, F259-F266 (2007).

[5] L. Dorst, D. Fontijne, S. Mann,, Geometric Algebra for Computer Science: An Object-Oriented Approach to Geometry, Morgan Kaufmann Publishers Inc., San Francisco, 2007.

[6] P. Feinsilver, U. Franz, R. Schott, Duality and multiplicative processes on quantum groups, J. Th. Prob., 10 (1997), 795-818.

[7] P. Feinsilver, J, Kocik, R. Schott, Representations of the Schrödinger algebra and Appell systems, Progress of Phys., 52 (2004), 343-359.

[8] P. Feinsilver, R. Schott, Appell systems on Lie groups, J. Th. Prob., 5 (1992), 251-281.

[9] P. Feinsilver, R. Schott, Algebraic Structures and Operator Calculus, Vol. 3: Representations of Lie Groups, Kluwer, Dordrecht, 1996.

[10] D. Fontijne, Efficient Implementation of Geometric Algebra (PhD. thesis), University of Amsterdam (2007).

[11] R.L. Hudson, A quantum mechanical central limit theorem for anticommuting observables, J. Appl. Probability, 10 502-509, (1973).

[12] T. Magulski, Ł. Orłowski, Geometric-algebra quantum-like algorithms: Simon's algorithm, Preprint quant-ph/0705.4289

[13] P. Lounesto, Clifford Algebras and Spinors, Cambridge University Press, Cambridge, 2001.

[14] R. Schott, G.S. Staples, Operator calculus and Appell systems on Clifford algebras, International Journal of Pure and Applied Mathematics, 31 (2006), 427-446.

[15] R. Schott, G.S. Staples, Nilpotent adjacency matrices, random graphs, and quantum random variables, J. Phys. A: Math. Theor., 41 155205, (2008).

[16] R. Schott, G.S. Staples. Random walks in Clifford algebras of arbitrary signature as walks on directed hypercubes, Markov Processes and Related Fields, 14 (2008), 515-542.

[17] R. Schott, G.S. Staples. Dynamic random walks in Clifford algebras, Advances in Pure and Applied Mathematics, 1 (2010), 81-115.

[18] R. Schott, G.S. Staples. Operator homology and cohomology in Clifford algebras, Cubo, A Mathematical Journal, 12 (2010), 299326.

[19] R. Schott, G.S. Staples. Zeons, lattices of partitions, and free probability, Comm. Stoch. Anal., 4 (2010), 311-334. 
[20] R. Schott, G.S. Staples. Nilpotent adjacency matrices and random graphs, Ars Combinatoria, 98 (2011), 225-239.

[21] G.S. Staples, Graph-theoretic approach to stochastic integrals with Clifford algebras, J. Theor. Prob., 20 257-274, (2007).

[22] G.S. Staples, A new adjacency matrix for finite graphs, , Advances in Applied Clifford Algebras, 18 979-991, (2008).

[23] W. von Waldenfels, An algebraic central limit theorem in the anticommuting case, Z. Wahrsch. verw. Gebiete, 42 135-140, (1978). 